\title{
Polypyrrole-incorporated conductive hyaluronic acid hydrogels
}

\author{
Jongcheol Yang ${ }^{\dagger}$, Goeun Choe ${ }^{\dagger}$, Sumi Yang, Hyerim Jo and Jae Young Lee
}

\begin{abstract}
Background: Hydrogels that possess hydrophilic and soft characteristics have been widely used in various biomedical applications, such as tissue engineering scaffolds and drug delivery. Conventional hydrogels are not electrically conductive and thus their electrical communication with biological systems is limited.

Method: To create electrically conductive hydrogels, we fabricated composite hydrogels of hyaluronic acid and polypyrrole. In particular, we synthesized and used pyrrole-hyaluronic acid-conjugates and further chemically polymerized polypyrrole with the conjugates for the production of conductive hydrogels that can display suitable mechanical and structural properties.

Results: Various characterization methods, using a rheometer, a scanning electron microscope, and an electrochemical analyzer, revealed that the PPy/HA hydrogels were soft and conductive with $\sim 3 \mathrm{kPa}$ Young's modulus and $\sim 7.3 \mathrm{mS} / \mathrm{cm}$ conductivity. Our preliminary in vitro culture studies showed that fibroblasts were well attached and grew on the conductive hydrogels.

Conclusion: These new conductive hydrogels will be greatly beneficial in fields of biomaterials in which electrical properties are important such as tissue engineering scaffolds and prosthetic devices.
\end{abstract}

Keywords: Polypyrrole, Hyaluronic Acid, Hydrogel, Conductive, Biomaterials

\section{Background}

Various types of hydrogels have gained attention as effective biomaterials for a last few decades. Hydrogels are three dimensional insoluble networks of hydrophilic polymer chains and swell in aqueous solutions. They can absorb a lot of water within their matrices. Hydrogels usually exhibit great biocompatibility, porosity, soft mechanical properties and ease in modification. Therefore, they have been extensively employed for various applications, such as tissue engineering scaffolds, tissue augments, and drug delivery vehicles. Although hydrogels have such good characteristics, hydrogels do not generally possess electrical conductivity [1]. Since electrical signals are involved in various biological events, such as tissue regeneration, muscle movement, cell communications, biomaterials that have electrical conductance have been fabricated to modulate cell/tissue

\footnotetext{
* Correspondence: jaeyounglee@gist.ac.kr

${ }^{\dagger}$ Equal contributors

School of Materials Science and Engineering, Gwangju Institute of Science and Engineering (GIST), Gwangju 500-712, Republic of Korea
}

responses for various applications, including tissue engineering scaffolds and bio-electrodes [2].

Recently, conductive polymers, such as polypyrrole (PPy), polyaniline, poly(3,4-ethylenedioxythiophene (PEDOT)), polythiophene, have been used as components for biomaterials and their applications where electrical signaling is important $[3,4]$ because they have good electrical characteristics and softer mechanical properties than metals [5-7]. Electrical signals can be efficiently transferred at the interfaces between cells and conductive substrates. For example, lower potentials can lead to more effective cellular modulation on conductive substrates compared to on nonconductive substrates allowing uses of lower electrical potentials. To take advantage of hydrogels and electrically conductive polymers for uses as biomaterials, electrically conductive hydrogel can be a promising platform. Conductive hydrogels typically consist of polymeric co-networks of structural polymers and electrically conductive polymers [3, $8-12]$. The conductive hydrogel scaffolds have potentials to achieve electrical communications between cells and stimulate cellular activity such as differentiation [13]. 
In our studies, we synthesized novel conductive hydrogels which are composed of pyrrole incorporated hyaluronic acid (HA) and PPy. HA is a non-sulfated glycosaminoglycan which is a major component of extracellular matrix. HA has been extensively utilized for a number of biomaterial applications due to its many advantages, such as biodegradability, biocompatibility, bioresorption, easy modification with many functional groups. It is also known as interaction with $\mathrm{CD} 44^{+}$cells such as normal stem cells (e.g., mesenchymal stem cells, neural stem cells, and hematopoietic stem cells) and cancer stem cells [14-22]. PPy is an organic conductive polymer and can be easily synthesized electrochemically or chemically. PPy displays inherent good conductivity, long-term stability, and biocompatibility [23], which have made PPy useful in numerous application such as biosensor, drug delivery system and other biomaterials [24-27]. In this study, covalent bond formation between $\mathrm{HA}$ and pyrrole were designed to enhance structural stability and uniformity of hydrogel. HA-pyrrole conjugates were first synthesized and polymerized together with pyrrole monomers to elongate PPy chains inside the composite hydrogels and also to form crosslinks between HA and PPy chains. Pyrrole monomer and oxidant concentrations were varied to produce different conductive hydrogels (i.e., PyHA-PPy). Additionally, fibroblasts were cultured on the produced PyHA-PPy hydrogels and its adhesion and growth were examined.

\section{Methods}

\section{Materials}

1-(2-cyanoethyl) pyrrole, lithium aluminum hydride, $\mathrm{N}$ (3-dimethylaminopropyl)-N'-ethylcarbodiimide hydrochloride (EDC), N-Hydroxysuccinimide (NHS), Ammonium persulfate (APS), and diethyl ether were provided from Sigma-Aldrich (St. Louis, MO, USA). Hyaluronic acid $\left(1 \times 10^{6} \mathrm{Da}\right)$ was kindly provided from the LG Life Science Ltd (South Korea). Dulbecco's modified Eagle's medium, fetal bovine serum (FBS), and Dulbecco's phosphate buffered saline (DPBS) were produced from Hyclone. Penicillin/Streptomycin and trypsin/EDTA were provided from Gibco (Gaithersburg, MD, USA). LIVE/DEAD viability/cytotoxicity kit and CMFDA cell tracker kit were purchased from Life Science Technology.

\section{Synthesis of $\mathrm{N}-(3$-aminopropyl)pyrrole}

$\mathrm{N}$-(3-aminopropyl) pyrrole was synthesized as previously described in the literature [27]. In brief, $0.02 \mathrm{~mol} \mathrm{1-2(2-}$ cyanoethyl)pyrrole was dissolved in anhydrous ethyl ether $(15 \mathrm{~mL})$. The 1-2(2-cyanoethyl)pyrrole solution was added into a $\mathrm{LiAlH}_{4}$ solution $(0.05 \mathrm{~mol}$ in anhydrous ethyl ether, $150 \mathrm{~mL}$ ). Then, the mixture was refluxed for $12 \mathrm{~h}$. After cooling, the excess hydride was precipitated to a solid form by the addition of the solutions in sequence of water $(1.7 \mathrm{~mL}), 15 \%(\mathrm{w} / \mathrm{v}) \mathrm{NaOH}$ $(1.7 \mathrm{~mL})$, and water $(5.1 \mathrm{~mL})$. The precipitations were filtered and the remained solvent was completely evaporated. ${ }^{1} \mathrm{H}$ NMR $\left(\mathrm{CDCl}_{3}\right)$ was obtained with this material was obtained. $1.9\left(\mathrm{~m}, 2 \mathrm{H}, \mathrm{CH}_{2}-2\right), 2.75(\mathrm{t}, 2 \mathrm{H}$, $\mathrm{CH}_{2}-3$ ), 4.0 (t, 2H, $\left.\mathrm{CH}_{2}-1\right), 6.1$ (d, $\left.2 \mathrm{H}, \mathrm{CH}-\beta\right), 6.65$ (d, $2 \mathrm{H}, \mathrm{CH}-\alpha)$.

\section{Preparation of pyrrole-hyaluronic acid conjugate (PyHA)} $0.1 \%(\mathrm{w} / \mathrm{v})$ hyaluronic acid sodium salt $\left(\mathrm{HA}, 1 \times 10^{6} \mathrm{Da}\right.$, medical use) solution was prepared by dissolving HA powder in deionized (DI) water. EDC $(1 \mathrm{mmol})$ and NHS $(1 \mathrm{mmol})$ were added in to the HA solution. Synthesized $\mathrm{N}$-(3-aminopropyl)pyrrole $1 \mathrm{mmol}$ was then added into the solution. After perfect dissolution $\mathrm{pH}$ of the solution $\mathrm{pH}$ was adjusted to 5.5 to enhance the reaction yield. After $20 \mathrm{~h}$ reaction in room temperature, the solution was dialyzed using (3.5 kDa MWCO, Spectrum laboratories) in DI water at room temperature for 6 days. The water was exchanged every $12 \mathrm{~h}$ for three days. The solution was freeze-dried after filtered with $0.22 \mu \mathrm{m}$ Bottom Top filter (Corning) and stored at $-20{ }^{\circ} \mathrm{C}$ until use. PyHA was characterized using ${ }^{1} \mathrm{H}$ NMR $\left(\mathrm{D}_{2} \mathrm{O}\right): 1.95$ (s, $\left.3 \mathrm{H}, \mathrm{C}(=\mathrm{O}) \mathrm{CH}_{3}\right), 6.2(\mathrm{~d}, 2 \mathrm{H}, \mathrm{CH}-\alpha-$ pyrrole), $6.7(\mathrm{~d}, 2 \mathrm{H}$, $\mathrm{CH}-\beta$-carbon). Degree of pyrrole subunit substitution is calculated via ${ }^{1} \mathrm{H}$ NMR from the ratio of the relative peak integrations of the pyrrole protons and HA methyl protons as $\sim 20 \%$.

\section{Fabrication of the PyHA-PPy hydrogels}

Polypyrrole/HA composite (PyHA-PPy) hydrogels were fabricated by polymerizing pyrrole within the preprepared PyHA hydrogels. To this end, oxidizing agent (i.e., APS) was added to induce PPy polymerization and crosslink the pyrrole moieties attached onto PyHA backbone. The previously synthesized PyHA was dissolved in DI water to have the final concentration $(1.0 \mathrm{w} / \mathrm{v} \%)$. Concentrations of pyrrole solutions (in DI water) were varied to be $0 \mathrm{mM}, 10 \mathrm{mM}, 25 \mathrm{mM}, 50 \mathrm{mM}$ and $100 \mathrm{mM}$, respectively. Then, the APS solution was prepared in the ranges from $50 \mathrm{mM}$ to $250 \mathrm{mM}$ of final concentrations. PyHA solution and pyrrole solution was mixed together and placed on ice to reach the solution temperature to $0{ }^{\circ} \mathrm{C}$. The APS solution is added into the solution containing PyHA and pyrrole. Then, the mixed solution is vigorously stirred for $30 \mathrm{~s}$ and placed between $2 \mathrm{~mm}$ gap for $2 \mathrm{~h}$ in room temperature. After a hydrogel was formed, the hydrogel sheet moved into the DPBS and incubated for 3 days by changing the DPBS for every $6 \mathrm{~h}$ to remove unreacted residual APS and pyrrole monomers inside the hydrogel. 


\section{Mechanical property measurement}

The mechanical property of the fabricated hydrogel was measured using a rheometer (KINEXUS). The hydrogel sheet was punched with $6 \mathrm{~mm}$ diameter matching with the geometry. The rheological measurement was taken with frequency sweep measurement from $0.1 \mathrm{~Hz}$ to $10 \mathrm{~Hz}$ with 0.04 strain. The Young's modulus was calculated from the obtained shear modulus at $1 \mathrm{~Hz}$ using the equation according to the literature.

\section{Electrical property measurement}

The electrical property of the hydrogel was measured using the 4-point probe system with Versastat. Before measurement, the hydrogels were washed with DPBS and dried in the air overnight. The dried hydrogels were swollen in DI water. Linear sweep voltammetry was applied and a bulk resistivity of the hydrogel was calculated as shown below.

$$
p=4.53 \times \mathrm{t} \times \frac{\mathrm{V}}{\mathrm{I}}
$$

where $\rho$ is the bulk resistivity and $t$ is the thickness of the substrate. The bulk resistivity could be calculated with the equation above. Next, the conductivity $(\sigma)$ was obtained from $1 / \rho$.

\section{In vitro fibroblast culture}

NIH 3 T3 fibroblasts were maintained in DMEM with $10 \% \mathrm{FBS}, 1 \%$ anti-anti with a $5 \% \mathrm{CO}_{2}$ at $37{ }^{\circ} \mathrm{C}$ humidified incubator. The medium was changed every 3 days to fresh medium. They were subculture when their confluency reached to $80 \%$. Subculture was performed with $0.05 \%$ trypsine- $0.53 \mathrm{mM}$ EDTA solution treatment for $5 \mathrm{~min}$ and cells were collected by centrifugation at $1200 \mathrm{rpm}, 5 \mathrm{~min}$. Cell numbers were counted using a hemocytometer. NIH-3 T3 was seeded as $5 \times 10^{4}$ cells/ $\mathrm{cm}^{2}$.

For the studies of cell growth on the PyHA-PPy hydrogels, the hydrogels were first washed for a week and punched with $8 \mathrm{~mm}$ diameter. And then washed with $70 \%$ of ethanol solution for $30 \mathrm{~min}$ and extensively washed with DPBS for 3 days, changing the DPBS every day. The NIH 3 T3 were seeded onto the hydrogels at a cell density of 50,000 cells $/ \mathrm{cm}^{2}$. The culture medium was added after $3 \mathrm{~h}$ in order to make the cells adhere onto the hydrogels. The medium was changed every 3 day. Cell viability was measured using the Live/dead viability/ cytotoxicity kit according to the protocol provided by the manufacturer. In brief, $5 \mu \mathrm{L}$ of $2 \mathrm{mM}$ calcein $\mathrm{AM}$ and $20 \mu \mathrm{L}$ of $4 \mathrm{mM}$ EthD-1 per $10 \mathrm{~mL}$ solution were used. After 10-15 min staining, the individual samples were washed with DPBS twice. Fixing was performed with $3.74 \%$ paraformaldehyde. Fluorescence images were acquired using a fluorescence microscope (Leica
DMI3000B). Live and dead cells were counted as green and red colors, respectively. Live cell numbers were counted from at least 5 randomly taken images.

\section{Results and discussion PyHA-PPy hydrogels fabrication}

The various PyHA-PPy hydrogels were fabricated with the different pyrrole concentrations (i.e., $0 \mathrm{mM}, 10 \mathrm{mM}$, $25 \mathrm{mM}, 50 \mathrm{mM}$, and $100 \mathrm{mM}$ ) as shown in Table 1 . The fabricated PyHA-PPy hydrogels were clean and not brittle. First, PyHA conjugates were chemically synthesized (Scheme 1). N-(3-aminopropyl) pyrrole was conjugated onto HA backbone using EDC/NHS chemistry. Hydrogel formation was expected to result from the oxidative coupling of the pyrrole moieties between HA chains and/or the coupling between the polymerized PPy chains and the conjugated pyrrole moieties presented on HA. The fabricated hydrogel in this manner could form stable covalent bonds between HA chains and the PPy chains, allowing for its structural stability. The fact that the hydrogel could be formed even without any additional pyrrole monomers in the presence of the APS suggests that the pyrrole moieties on PyHA were associated to form covalent bonds. Furthermore, with an increase in a pyrrole monomer concentration, PPy contents in the PyHA-PPy hydrogels appeared to increase, which could consequently increase stiffness and electrical conductivity. In our studies, as the pyrrole monomer and oxidant concentrations increased, the resultant hydrogels exhibited darker color, which indicates that the added pyrrole monomers were oxidized into PPy with the PyHA hydrogels. As mentioned above, simple mixing of the PyHA solutions and APS without any additional pyrrole monomers could lead to hydrogel formation (Fig. 1a). It should be noted that the sizes of hydrogels decreased after the PPy polymerization with oxidants. These size decreases of the hydrogels were more distinct for the samples synthesized at higher pyrrole monomer concentrations (higher PPy contents). These results may result from the high entanglement degrees due to more chain units and/or decreases in hydrophilicity due to increases in less hydrophilic PPy portions.

\section{PyHA-PPy hydrogel morphologies}

Internal structures of the hydrogels were examined by SEM. All fabricated hydrogels showed the microporous structures inside the hydrogel (Fig. 1b). The pore sizes appeared to be in the ranges of $10 \mu \mathrm{m}$. Interestingly, web-like structures with globular shape with size less than $100 \mathrm{~nm}$ were observed when the PPy portions were high in the hydrogels. These PPy structures were prominently observed from PyHA-PPy100 hydrogels and PyHA-PPy10 hydrogels. The conventional PPy was 
Table 1 The names of various PyHA-PPy hydrogels and their synthetic conditions

\begin{tabular}{llllll}
\hline Sample Names & PyHA-PPy 0 & PyHA-PPy 10 & PyHA-PPy 25 & PyHA-PPy 50 & PyHA-PPy 100 \\
\hline PyHA solution & $1.0 \%$ & $1.0 \%$ & $1.0 \%$ & $1.0 \%$ & $1.0 \%$ \\
Pyrrole & $0 \mathrm{mM}$ & $10 \mathrm{mM}$ & $25 \mathrm{mM}$ & $50 \mathrm{mM}$ & $100 \mathrm{mM}$ \\
APS & $50 \mathrm{mM}$ & $75 \mathrm{mM}$ & $100 \mathrm{mM}$ & $125 \mathrm{mM}$ & $100 \mathrm{mM}$ \\
\hline
\end{tabular}

All concentrations were described as the final concentrations after mixing all components

reported to have sphere-like structures or web-like globular shape structures when polymerized chemical oxidants. Observed web-like PPy morphologies imply that PPy chains grew inside the hydrogels.

\section{Characterization of PyHA-PPy hydrogels}

The modulus was measured using an oscillatory rheometer in a frequency sweep mode. The moduli of the hydrogels increased with increases in the added pyrrole monomer concentrations by $50 \mathrm{mM}$ pyrrole. Results indicate that PPy in the hydrogel might contribute the enhancement of the modulus of the hydrogels. The Young's modulus was in a range from $0.6 \mathrm{kPa}$ to $3 \mathrm{kPa}$. However, the modulus decreased when the pyrrole concentration was above $50 \mathrm{mM}$ (Fig. 2a). PyHA-PPy 100 hydrogels did not follow the general trend that the increases in the PPy portions inside the hydrogels result in the increases of both the stiffness and electrical properties. It may be due to heterogeneous composite formation by heterogeneous PPy incorporation. Too high pyrrole concentrations and oxidants might lead to too fast reaction rate inside the hydrogel or in the polymerizing solution (outside the hydrogel). Since free pyrrole monomers can be oxidized more readily than the pyrrole moieties attached on PyHA, PPy formed in the solution not in the hydrogels and deposited on the surfaces of the hydrogels. Also, the pyrrole groups on the PyHA might not be sufficiently associated with PPy polymerization in PyHA-PPy 100 hydrogel, resulting in insufficient covalent bond formation in PyHA-PPy and poor stability of mechanical and electrical properties.

The electrical conductivity of the hydrogels was measured (Fig. 2b). There were few reports about the measurement of conductivity of conductive hydrogels and therefore it is difficult to directly compare the results. Hur et al. reported that the PPy agarose based hydrogel showed the conductivity in the order of $10^{-1} \mathrm{~S} / \mathrm{cm}[28]$. Shi et al. fabricated the cellulose/polypyrrole composite
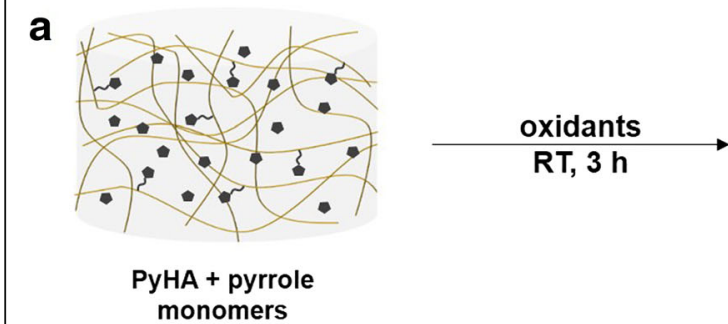

yHA + pyrrole
monomers

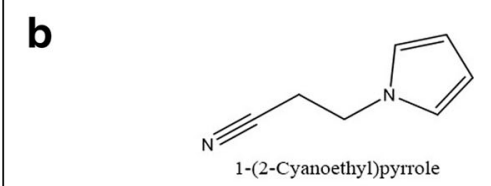

Synthesis of $\mathbf{N}$-(aminopropyl)pyrrole

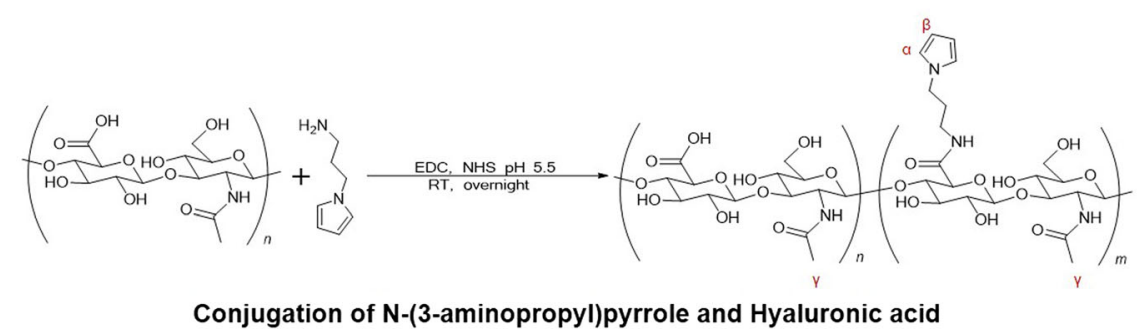

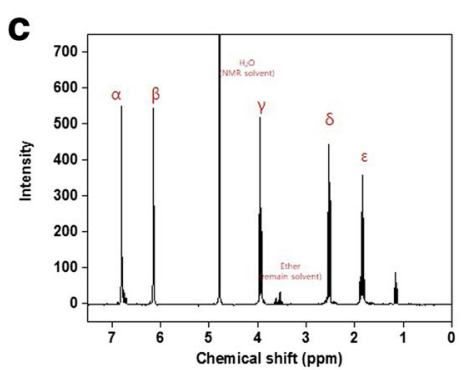

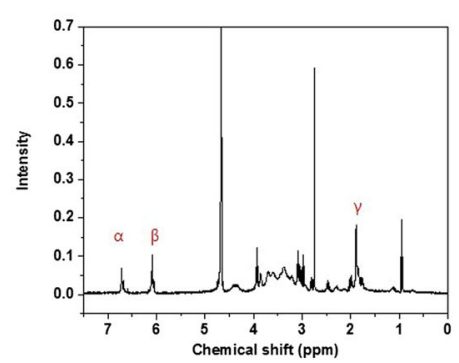

Scheme 1 (a) Scheme of PyHA-PPy hydrogels synthesis. (b) Chemistry of N-(3-aminopropyl)pyrrole synthesis and pyrrole-HA conjugate synthesis (c) $\mathrm{H}^{1} \mathrm{NMR}$ spectra of $\mathrm{N}$-(3-aminopropyl)pyrrole (top) and PyHA conjugate (bottom) 


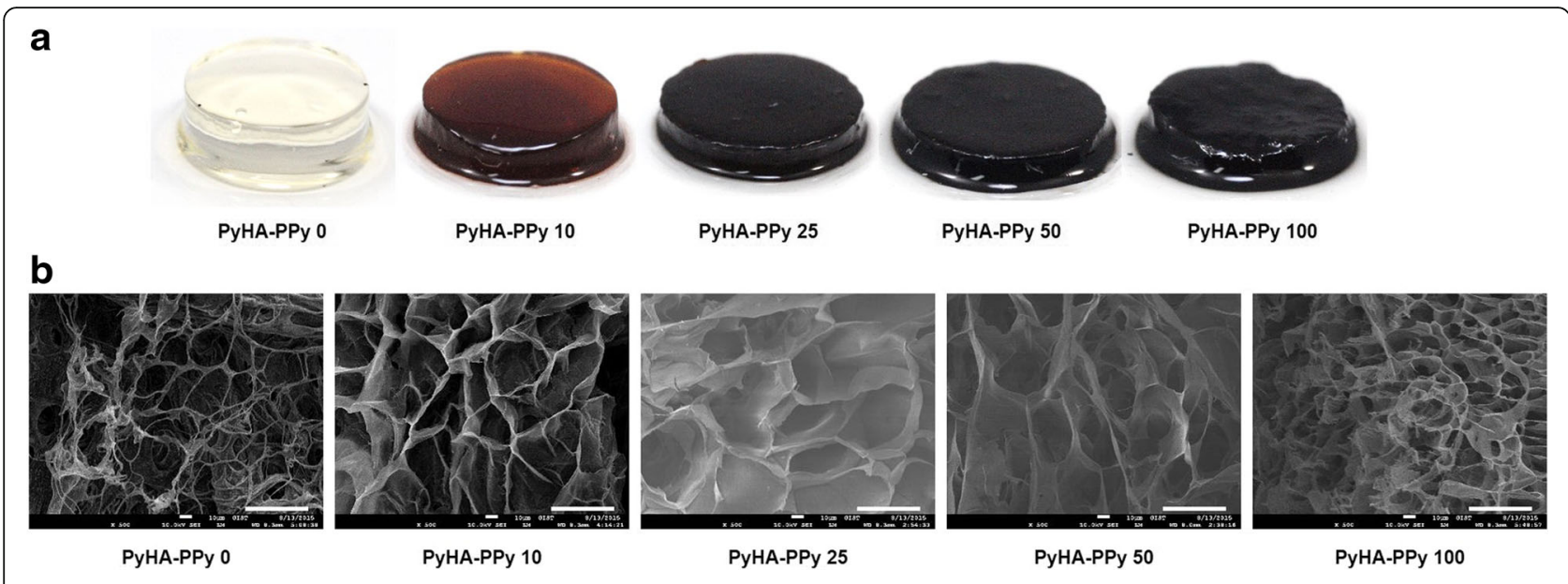

Fig. 1 a Photographs of the various PyHA-PPy hydrogels. b SEM images of the PyHA-PPy hydrogels. Scale bars are $50 \mu$ m

aerogels and found their conductivity were approximately $0.08 \mathrm{~S} / \mathrm{cm}$ [26]. Our hydrogels made of PPy and PyHA had the conductivities in a range of $10^{-3}-10^{-2} \mathrm{~S} /$ $\mathrm{cm}$. The conductivity of the hydrogels was increased with the amount of the polypyrrole inside the hydrogel increased until the pyrrole concentration in the polymerizing solution was $50 \mathrm{mM}$. The highest conductivity, $7.27 \mathrm{mS} / \mathrm{cm}$ was measured from the PyHA-PPy 50 hydrogel that also showed the highest stiffness. This observation might be associated with the similar reasons with the weaker mechanical property PyHA-PPy 100 hydrogel than PyHA-PPy 50, which include structural instability and non-uniformity. For example, in the case of the PyHA-PPy 100 hydrogels, too fast reactions led to the local aggregation of PPy on the surface of the hydrogels, which had limited effects on the conductivity.

\section{In vitro study}

Because PyHA-PPy 50 showed the greatest electrical properties with appropriate soft characteristics $(\sim 3 \mathrm{kPa}$
Young's modulus) among differently fabricated PyHAPPy hydrogels, we selected PyHA-PPy 50 hydrogels for further in vitro studies (Fig. 3). These mechanical and electrical properties of the fabricated PyHA-PPy hydrogels appear to be suitable for the applications where electrical signals were needed in soft environments. We examined the cell adhesion and proliferation on the PyHA-PPy 50 substrates using widely used murine $3 \mathrm{~T} 3$ fibroblasts. First, we attempted to culture the cells on the substrates without treatment of any cell-adhesive molecules. Cells were well-attached on the PyHA-PPy $50 \mathrm{mM}$, indicating the hydrogels' ability to allow cell adhesion even without any pre-coating. Since HA is generally non-cell adhesive, we speculate that PPy portions might play important roles in promoting cell adhesion. At day 1 , about 90 cells $/ \mathrm{mm}^{2}$ were attached. Adhered cells showed spherical morphology. Further incubation allowed cell proliferation. At day 5, the number of the cells was increased by approximately 6 times $\left(545\right.$ cells $\left./ \mathrm{mm}^{2}\right)$. In particular, cells showed stretched morphologies, indicating
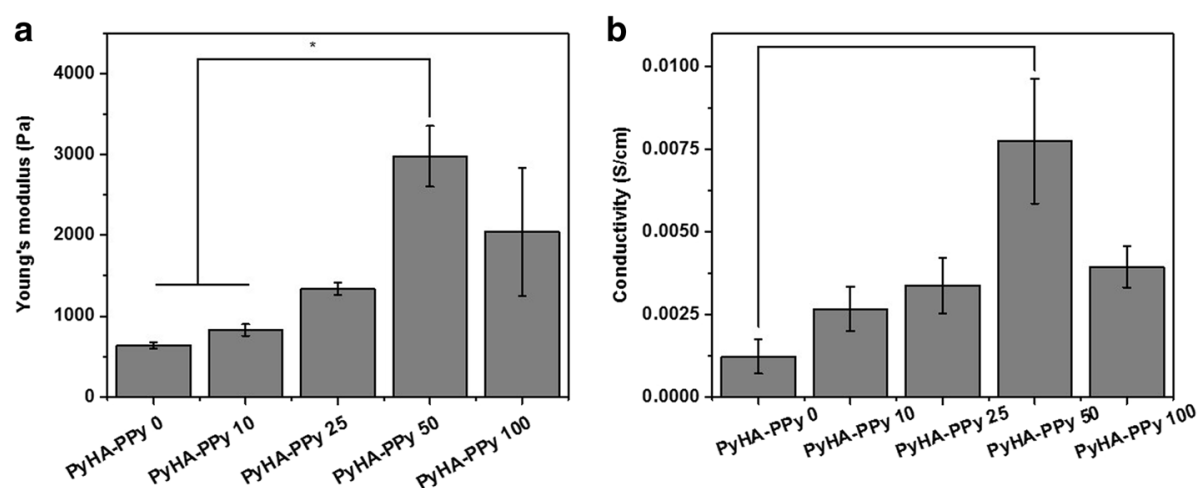

Fig. 2 Mechanical and electrical properties of the PyHA-PPy hydrogels. a Young's modulus of the fabricated hydrogels. b Conductivity of the fabricated hydrogels. Averages and standard deviations were plotted $(n=3)$. Statistical difference was assessed using the student $t$-test and statistical significance was denoted with an asterisk $(p<0.05)$ 

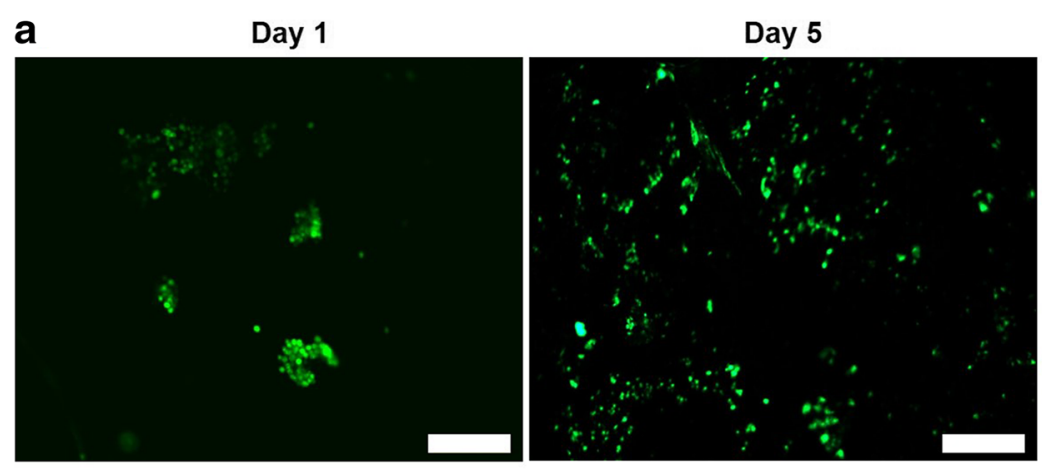

b

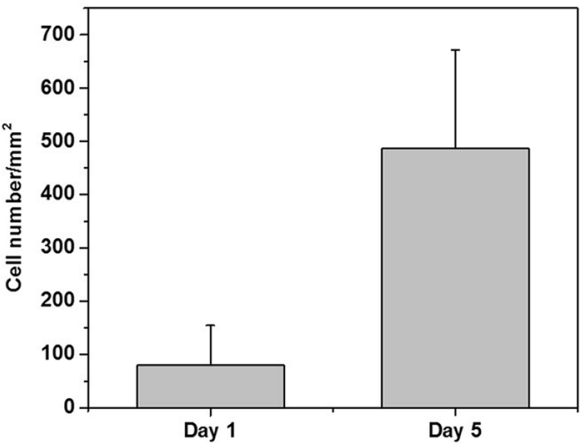

Fig. 3 a Live/dead staining images of the 3 T3 cells on PyHA-PPy 50 hydrogels at day 1 and 5, respectively. $\mathbf{b}$ A plot of the attached cell numbers on the PyHA-PPy 50 hydrogel at day 1 and 5. Averages and standard deviations were plotted $(n=5)$. Scale bars are $200 \mu \mathrm{m}$

viable cells on the PyHA-PPy. Hence, cells were highly viable on PyHA-PPY $50 \mathrm{mM}$ hydrogel at both days. As results, our PyHA-PPy hydrogels can support cell adhesion and proliferation. Further studies will be needed for the cultivation of other types of cells, such as stem cells and neural cells for specific tissue engineering scaffold applications. Also, studies on the effects of electrical stimulation of cells via our conductive hydrogels will be needed to clearly demonstrate the benefits of conductive hydrogels.

\section{Conclusion}

We fabricated PyHA-PPy hydrogels via covalent bond formation and PPY polymerization. These hydrogels were soft, porous, structurally stable and electrically conductive. As the added pyrrole concentration increased, fabricated hydrogels showed darker color, higher mechanical and electrical properties. Among various conductive hydrogels, the PyHA-PPy 50 showed $\mathrm{mM}$ showed the highest $7.3 \mathrm{mS} / \mathrm{cm}$ with softness $(\sim 3$ $\mathrm{kPa}$ Young's modulus). In addition, in vitro study showed good cell adhesion and proliferation on the PyHA-PPy 50 substrates. Our new conductive hydrogels will be useful in tissue engineering field which needs electrical stimulation and mechanical softness.

\section{Abbreviations}

APS: Ammonium persulfate; CMFDA: 5-chloromethylfluorescein diacetate; DI: De-ionized; DPBS: Dulbeco's phosphate buffered saline; FBS: Fetal bovine serum; HA: Hyaluronic acid; PPY: Polypyrrole; PyHA: Pyrrole-conjugated hyaluronic acid; PyHA-PPy: Polypyrrole-incorporated PyHA hydrogel

\section{Acknowledgement}

Not applicable.

\section{Funding}

This research was supported by the Basic Science Research Program (2013R1A1A1012179) through the National Research Foundation of Korea (NRF) funded by the Ministry of Science, ICT \& Future Planning. Also, this research was supported by a grant of the Korea Health Technology R\&D Project through the Korea Health Industry Development Institute (KHIDI), funded by the Ministry of Health \& Welfare (HI14C3484), Republic of Korea.
Availability of data and materials

The data sets and materials supporting the results of this article are included in the article.

\section{Authors' contributions}

$J \mathrm{YL}$ designed the concept and experiments and helped to draft the manuscript. JCY synthesized and characterized materials, and drafted the manuscript. GEC performed the in vitro experiment and drafted the manuscript. SMY participated in the design of experiment. HRJ performed SEM imaging. All authors read and approved the final manuscript.

\section{Competing interests}

The authors declare that they have no competing interests.

\section{Consent for publication}

The manuscript has been submitted with the consent of all authors and data of any other person not included.

Ethics approval and consent to participate

Manuscript does not include human ethics value. Hence no consent is needed.

Received: 28 July 2016 Accepted: 14 September 2016

Published online: 29 September 2016

\section{References}

1. O'brien FJ. Biomaterials \& scaffolds for tissue engineering. Mater Today. 2011;14:88-95.

2. Hardy JG, Lee JY, Schmidt CE. Biomimetic conducting polymer-based tissue scaffolds. Curr Opin Biotechnol. 2013;24:847-54.

3. Balint R, Cassidy NJ, Cartmell SH. Conductive polymers: Towards a smart biomaterial for tissue engineering. Acta Biomater. 2014;10:2341-53.

4. Pires F, Ferreira Q, Rodrigues CA, Morgado J, Ferreira FC. Neural stem cell differentiation by electrical stimulation using a cross-linked PEDOT substrate: expanding the use of biocompatible conjugated conductive polymers for neural tissue engineering. Biochim Biophys Acta BBA-Gen Subj. 2015;1850: 1158-68.

5. Green RA, Baek S, Poole-Warren LA, Martens PJ. Conducting polymerhydrogels for medical electrode applications. Sci Technol Adv Mater. 2016.

6. Guimard NK, Gomez N, Schmidt CE. Conducting polymers in biomedical engineering. Prog Polym Sci. 2007;32:876-921.

7. Bendrea A-D, Cianga L, Cianga I. Review paper: progress in the field of conducting polymers for tissue engineering applications. J Biomater Appl. 2011;26:3-84

8. Guiseppi-Elie A. Electroconductive hydrogels: Synthesis, characterization and biomedical applications. Biomaterials. 2010;31:2701-16.

9. Qu B, Chen C, Qian L, Xiao H, He B. Facile preparation of conductive composite hydrogels based on sodium alginate and graphite. Mater Lett. 2014;137:106-9.

10. Peng $R$, Yu Y, Chen S, Yang Y, Tang Y. Conductive nanocomposite hydrogels with self-healing property. RSC Adv. 2014;4:35149-55. 
11. Guarino V, Alvarez-Perez MA, Borriello A, Napolitano T, Ambrosio L. Conductive PANi/PEGDA macroporous hydrogels for nerve regeneration. Adv Healthc Mater. 2013;2:218-27.

12. Kim D, Abidian M, Martin DC. Conducting polymers grown in hydrogel scaffolds coated on neural prosthetic devices. J Biomed Mater Res A. 2004; 71:577-85.

13. Thrivikraman G, Madras G, Basu B. Intermittent electrical stimuli for guidance of human mesenchymal stem cell lineage commitment towards neural-like cells on electroconductive substrates. Biomaterials. 2014;35:6219-35.

14. Collins MN, Birkinshaw C. Hyaluronic acid based scaffolds for tissue engineering -A review. Carbohydr Polym. 2013:92:1262-79.

15. Prestwich GD. Hyaluronic acid-based clinical biomaterials derived for cell and molecule delivery in regenerative medicine. J Controlled Release. 2011; 155:193-9.

16. Segura T, Anderson BC, Chung PH, Webber RE, Shull KR, Shea LD. Crosslinked hyaluronic acid hydrogels: a strategy to functionalize and pattern. Biomaterials. 2005;26:359-71.

17. Kogan G, Šoltés L, Stern R, Gemeiner P. Hyaluronic acid: a natural biopolymer with a broad range of biomedical and industrial applications. Biotechnol Lett. 2007;29:17-25.

18. Yoo HS, Lee EA, Yoon JJ, Park TG. Hyaluronic acid modified biodegradable scaffolds for cartilage tissue engineering. Biomaterials. 2005;26:1925-33.

19. Solis MA, Chen Y-H, Wong TY, Bittencourt VZ, Lin Y-C, Huang LL. Hyaluronan regulates cell behavior: a potential niche matrix for stem cells. Biochem Res Int. 2012;2012.

20. Zhu H, Mitsuhashi N, Klein A, Barsky LW, Weinberg K, Barr ML, et al. The role of the hyaluronan receptor CD44 in mesenchymal stem cell migration in the extracellular matrix. Stem Cells. 2006;24:928-35.

21. Miyake K, Underhill CB, Lesley J, Kincade PW. Hyaluronate can function as a cell adhesion molecule and CD44 participates in hyaluronate recognition. J Exp Med. 1990;172:69-75.

22. Lei Y, Gojgini S, Lam J, Segura T. The spreading, migration and proliferation of mouse mesenchymal stem cells cultured inside hyaluronic acid hydrogels. Biomaterials. 2011;32:39-47.

23. Ateh D, Navsaria H, Vadgama P. Polypyrrole-based conducting polymers and interactions with biological tissues. J R Soc Interface. 2006;3:741-52.

24. Stewart E, Kobayashi NR, Higgins MJ, Quigley AF, Jamali S, Moulton SE, et al. Electrical stimulation using conductive polymer polypyrrole promotes differentiation of human neural stem cells: a biocompatible platform for translational neural tissue engineering. Tissue Eng Part C Methods. 2014;21: 385-93.

25. Ahuja T, Mir IA, Kumar D. Biomolecular immobilization on conducting polymers for biosensing applications. Biomaterials. 2007;28:791-805.

26. Shi Z, Gao H, Feng J, Ding B, Cao X, Kuga S, et al. In situ synthesis of robust conductive cellulose/polypyrrole composite aerogels and their potential application in nerve regeneration. Angew Chem Int Ed. 2014;53:5380-4.

27. Abu-Rabeah K, Polyak B, lonescu RE, Cosnier S, Marks RS. Synthesis and characterization of a pyrrole-alginate conjugate and its application in a biosensor construction. Biomacromolecules. 2005;6:3313-8.

28. Hur J, Im K, Kim SW, Kim J, Chung D-Y, Kim T-H, et al. Polypyrrole/agarosebased electronically conductive and reversibly restorable hydrogel. ACS Nano. 2014:8:10066-76.

\section{Submit your next manuscript to BioMed Central and we will help you at every step:}

- We accept pre-submission inquiries

- Our selector tool helps you to find the most relevant journal

- We provide round the clock customer support

- Convenient online submission

- Thorough peer review

- Inclusion in PubMed and all major indexing services

- Maximum visibility for your research

Submit your manuscript at www biomedcentral.com/submit 